\title{
DISTINTAS LECTURAS DE LA PREGUNTA COMO MEDIACIÓN DIDÁCTICA PARA LA TRADUCCIÓN DE SABERES EN LA EDUCACIÓN SUPERIOR O ACERCA DE UN ESTADO EN CUESTIÓN
}

\author{
Lina María Grisales Franco*; Elvia María González Agudelo**
}

Recebido: 27 jul. $2012 \quad$ Aprovado: 6 nov. 2012

* Universidad de Antioquia. Medellín, Colombia. Contato: lgrisales2610@gmail.com

** Universidad de Antioquia. Medellín, Colombia. Contato: egonzal@ayura.udea.edu.co

Resumen: A partir de una búsqueda documental en el Sistema de Bibliotecas de la Universidad de Antioquia, bibliotecas digitales, revistas y en bases de datos especializadas en educación, se seleccionaron 26 investigaciones desarrolladas entre 1997 y 2007 para tener una aproximación a lo que actualmente se está investigando en relación con la pregunta en la educación superior. Se identificaron tres perspectivas en las que se mueve la investigación respecto a la pregunta. La primera, se refiere a los tipos de preguntas que se utilizan frecuentemente en la enseñanza superior. La segunda, sobre estrategias didácticas que se fundamentan en la pregunta. Y la tercera, acerca de la pregunta para posibilitar la comprensión y el desarrollo del pensamiento crítico.

Palabras clave: Pregunta. Mediación didáctica. Didáctica universitaria. Educación superior.

\section{DIFFERENT READINGS OF THE QUESTION AS DIDACTIC MEDIATION FOR THE TRANSLATION OF KNOWLEDGE IN HIGHER EDUCATION OR FOR A STATE IN QUESTION}

Abstract: Based on a literature search in the Library System of the University of Antioquia, digital libraries, journals and specialized databases in education, 26 research studies carried out between 1997 and 2007 were selected to have an approach as to what is currently being researched with regard to the question in higher education. Three perspectives in which research is moving relative to the question were identified. The first refers to the types of questions that are frequently used in higher education. The second, on teaching strategies that are based on the question. And third, about the question to enable the understanding and development of critical thinking.

Keywords: Question. Educational mediation. University teaching. Higher education.

\section{DIFERENTES LEITURAS DA PERGUNTA COMO MEDIAÇÃO DIDÁTICA PARAA TRADUCCÃO DE SABERES NA EDUCAÇÃO SUPERIOR OU A RESPEITO DE UM ESTADO EM QUESTÃO}

Resumo: A partir de uma busca documental no Sistema de Bibliotecas da Universidade de Antioquia, bibliotecas digitais, revistas e em bases de dados especializadas em educação, foram selecionados 26 pesquisas desenvolvidas entre 1997 e 2007 para obter uma aproximação ao que atualmente se está investigando em relação com a pergunta na educação superior. Foram identificadas três perspectivas nas quais se move a pesquisa com respeito à pergunta. A primeira se refere aos tipos de perguntas que se utilizam frequentemente no ensino superior. A segunda sobre estratégias didáticas que se fundamenta na pergunta. E a terceira, acerca da pergunta para possibilitar a compreensão e o desenvolvimento do pensamento crítico.

Palavras-chave: Pergunta. Mediação didática. Didática universitária. Educação superior. 


\section{INTRODUCCIÓN 1}

El siguiente artículo pretende dar cuenta del estado actual en que se ubica la investigación en didáctica universitaria, particularmente en lo relacionado a la pregunta como mediación didáctica para la traducción de los saberes. El texto se construyó mediante la búsqueda, selección y comparación de investigaciones que se realizaron entre 1997 y 2007 sobre la pregunta como mediación didáctica para la traducción de los saberes a nivel superior y utilizando el proceso hermenéutico de análisis, reflexión, comparación, comprensión, interpretación y síntesis, se logró hacer un acercamiento al presente de la cuestión.

La pregunta ¿la capacidad del docente de preguntarse y preguntar se constituye en una mediación didáctica para traducir los saberes en la educación superior? abrió el camino a la comprensión de un entramado de conocimientos sobre el tema. De modo que los conceptos que se buscaron fueron, en tanto partes, traducción, mediación y pregunta y en tanto todo, didáctica universitaria, la cual representa la expectativa de sentido de la búsqueda, selección y comparación de las investigaciones.

\section{BÚSQUEDA DOCUMENTAL}

Se realizó una búsqueda documental para recuperar el conocimiento producido entre 1997 y 2007, que desde el área de la educación está relacionado con la pregunta como una posibilidad para la traducción de los saberes a nivel superior. Se buscaron materiales como artículos de revistas, libros producto de investigación, tesis, monografías e informes de investigación. No se tuvieron en cuenta otros materiales como los ensayos por tratarse de reflexiones sobre un tema. El interés en este momento es conocer cómo ha sido desarrollado epistemológica, teórica y metodológicamente el tema de la pregunta para la traducción de los saberes y se considera que las investigaciones y sus resultados son los materiales más apropiados ya que aportan elementos clave para hacer esta construcción.

La consulta de los materiales se realizó en el Sistema de Bibliotecas de la Universidad de Antioquia, bibliotecas digitales como la Biblioteca Digital de la Organización de Estados Iberoamericanos y Metabiblioteca, en revistas en línea especializadas en el discurso educativo y en bases de datos especializa-

1 El artículo es parte de la tesis doctoral "Desde la pregunta en tanto mediación hacia la traducción como un principio didáctico en la educación superior", en la cual se comprende al docente universitario como un traductor de saberes, quien al momento de enseñar comunica el sentido del saber que enseña por medio de las secuencias de preguntas que se hacen expresas en clase. 
das en educación: unas disponibles en el Sistema de Bibliotecas de la Universidad de Antioquia y otras de acceso libre como Latindex, Google Académico, Redalyc y AERA. Adicionalmente se consultaron los trabajos realizados en el Grupo de Investigación Didáctica de la Educación Superior (DIDES) de la Universidad de Antioquia.

Antes de ubicar los materiales se definió el perfil de búsqueda teniendo en cuenta los conceptos que son desarrollados en la investigación y luego con la ayuda del Tesauro de la $\mathrm{UNESCO}^{2}$ se definieron los descriptores, es decir, los términos que mejor representan el contenido de cada uno de los conceptos, para de esta forma facilitar el acceso a la información. Por ser la UNESCO una autoridad internacional en los temas de educación, se piensa que este Tesauro indica la mejor palabra. En la tabla 1 se señalan los descriptores utilizados.

Tabla 1 - Descriptores utilizados en la búsqueda de los materiales para la construcción del estado en cuestión

\begin{tabular}{cccc}
\hline $\begin{array}{c}\text { Conceptos o } \\
\text { palabras clave } \\
\begin{array}{c}\text { (sugeridos por la } \\
\text { investigación) }\end{array}\end{array}$ & $\begin{array}{c}\text { Palabras clave } \\
\text { en Inglés }\end{array}$ & $\begin{array}{c}\text { Descriptores } \\
\text { (sugeridos por } \\
\text { el Tesauro) }\end{array}$ & $\begin{array}{c}\text { Descriptores } \\
\text { en Inglés }\end{array}$ \\
\hline Traducción & Translation & Traducción & Translation \\
\hline Pregunta & Question & $\begin{array}{c}\text { Interrogación } \\
\text { (método pedagógico) }\end{array}$ & $\begin{array}{c}\text { Questioning } \\
\text { (teaching method) }\end{array}$ \\
\hline Mediación & Mediation & Recursos educacionales & $\begin{array}{c}\text { Educational } \\
\text { resources }\end{array}$ \\
\cline { 2 - 4 } & & $\begin{array}{c}\text { Material didáctico } \\
\text { (material pedagógico, } \\
\text { medio pedagógico) }\end{array}$ & $\begin{array}{c}\text { Teaching materials } \\
\text { Didáctica }\end{array}$ \\
\cline { 2 - 4 } & Didactic & Técnica didáctica & $\begin{array}{c}\text { Classroom } \\
\text { techniques }\end{array}$ \\
\hline Educación superior & Higher Education & Enseñanza superior & Higher education \\
\cline { 2 - 4 } & Enseñanza de las ciencias & Science education \\
\hline
\end{tabular}

En total se revisaron 112 revistas, 196 trabajos de especialización y maestría, 7 tesis doctorales y 13 bases de datos. A partir del título, del resumen y de las palabras clave de los materiales se presumió la pertinencia de estos trabajos para la construcción del estado en cuestión. Si bien la búsqueda de los materiales se realizó en diversas fuentes, las bases de datos resultan ser una fuente de consulta importante por encontrarse allí gran número de publicacio-

2 Disponible en: <http://bmedica.udea.edu.co/moodle/mod/resource/view.php?id=1924> 
nes a nivel mundial. Es así, como se recuperaron 1'390.979 documentos relacionados con traducción, pregunta, mediación, didáctica y educación superior.

Al momento de ensayar combinaciones de los descriptores, el número de documentos se reduce considerablemente. Si bien, 1'390.979 registros relacionados con los conceptos de la investigación son una buena cantidad de material para la construcción del estado en cuestión, sólo el 0.001\% (16 documentos) responden a los intereses particulares, no porque se trate explícitamente de investigaciones en torno a la pregunta como mediación didáctica para la traducción de los saberes a nivel superior, sino porque se consideran que estas investigaciones aportan elementos importantes para comprender el trabajo investigativo que se ha adelantado en los últimos años (entre 1997 y 2007) en torno a la pregunta en el contexto de la educación superior. De las otras fuentes de búsqueda (diferentes a las bases de datos) se seleccionaron 10 documentos.

\section{SELECCIÓN DE LAS INVESTIGACIONES}

Cabe anotar que no se encontraron investigaciones en torno al uso de la pregunta como mediación didáctica para la traducción de los saberes a nivel superior. Sin embargo, se seleccionaron 26 investigaciones sobre la pregunta como estrategia didáctica por considerar que en estos trabajos se pueden encontrar indicios para pensar la pregunta como una posibilidad para traducir los saberes a nivel superior.

Estas investigaciones fueron leídas, analizadas, reflexionadas, comprendidas e interpretadas para establecer una comparación entre ellas y llegar a una síntesis que da cuenta del estado en cuestión. Para sistematizar la información bibliográfica acopiada se realizaron fichas referenciales.

De las 26 investigaciones seleccionadas 16 corresponden a artículos de revistas, 6 a trabajos de investigación, 2 a monografías, 1 a un informe de investigación de maestría y 1 a una tesis doctoral. La mitad de las investigaciones están publicadas en inglés y la otra mitad en español, sin observarse diferencias en los años de publicación. Sin embargo, no se recuperaron registros del año 2000 ni del 2002.

La mayoría de las investigaciones fueron recuperadas de bases de datos. Solo 2 se obtuvieron del archivo del grupo de investigación DIDES, 3 del Centro de Documentación de la Facultad de Educación de la Universidad de Antioquia y 1 de la biblioteca digital de la OEI. Por esta misma razón, la mayoría de éstas están en medio digital. 


\section{CONOCIMIENTO EN TORNO A LA PREGUNTA COMO MEDIACIÓN DIDÁCTICA PARA LA TRADUCCIÓN DE SABERES A NIVEL SUPERIOR}

En el ámbito de la enseñanza universitaria, la pregunta ha sido estudiada básicamente desde tres perspectivas. La más generalizada, tiene que ver con los tipos de preguntas y sus efectos en el aprendizaje. Esta perspectiva, va muy de la mano de una segunda, en la cual se incluyen los estudios que abordan la pregunta desde una mirada estratégica, es decir, estudian estrategias didácticas basadas en ella. Y también se encuentran investigaciones que estudian la pregunta en el marco del pensamiento crítico, siendo ésta una tercera línea de trabajo sobre el tema. Si bien, se identifican estas tres proyecciones en el abordaje de la pregunta, es difícil llegar a establecer unos límites claros entre estas categorías, ya que se influyen mutuamente. El hecho, es que todos son caminos que conducen a una mejor comprensión de la pregunta.

De la pregunta como mediación didáctica para la traducción de saberes en la educación superior no se tienen referencias específicas, sin embargo, en los trabajos seleccionados se identifican algunas pistas que conducen a los intereses particulares de la investigación. Al parecer en la pregunta subyacen relaciones entre el contenido, el método de enseñanza y la traducción de saberes.

Antes de entrar a analizar los trabajos que se ubican dentro de cada perspectiva, es interesante mirar cómo ha sido desarrollado el concepto pregunta en estas investigaciones. González (1997) plantea que la estrategia de la pregunta es una propuesta didáctica de acercamiento al conocimiento a través del diálogo dentro de un proceso de comunicación entre docentes y discentes que conduce a la comprensión y a la interpretación de textos. De una manera complementaria Morata Sebastián y Rodríguez Sánchez (1997) trabajan el concepto interrogación como el vehículo más apropiado para establecer un proceso comunicativo bidireccional entre dos o más sujetos. Por su parte, Abrandt Dahlgren y Öberg (2001) respaldan su trabajo en que las preguntas permiten aprender un contenido y ser consciente del proceso de adquisición de éste. El-Koumy (1997) desarrolla el concepto de self-questioning (o preguntar-se) como una estrategia que le da al estudiante el control de su aprendizaje, ganando independencia en él. En este mismo sentido, Valera Villegas y Madriz (1998) afirman que la pregunta constituye un proceso mediador hacia la formación de un hombre con actitud crítica.

Se comprende de estos aportes que los sentidos que guarda el concepto pregunta son el de medio, en tanto estrategia y vehículo; el de mediación, en tanto posibilita el acercamiento al conocimiento, el aprendizaje de un conteni- 
do, la conciencia del aprendizaje, la formación y el pensamiento crítico -con lo que posiblemente se establece un vínculo entre la pregunta, el contenido y el método de enseñanza, en tanto la pregunta es una mediación para acercar a un conocimiento-; y el de comunicación, en tanto abre el diálogo entre profesores y estudiantes, para compartir significados generando de este modo, procesos de interpretación que posibilitan llegar a acuerdos.

Esta conceptualización brinda un marco de referencia dentro del cual se mueve la investigación en torno a la pregunta y de esta manera se puede tener una mejor comprensión de la temática y evidenciar relaciones útiles para la construcción del estado en cuestión. Si bien, en esta conceptualización no se considera la traducción, se sospecha que el acercamiento al conocimiento, el aprendizaje de un contenido e incluso el pensamiento crítico están mediados por procesos de traducción, en la medida en que la traducción es un proceso que acerca al autor y al lector y a la vez, es una síntesis para expresar la comprensión del sentido de un texto.

En cuanto a los estudios que se ocupan de los tipos de preguntas y sus efectos en el aprendizaje (la primera perspectiva), se plantea que el tipo y la cantidad de preguntas que formulan profesores y estudiantes tienen implicaciones en el proceso de enseñanza y de aprendizaje a nivel superior. Por ejemplo, El-Koumy (1997) afirma que en los grupos donde el profesor y el estudiante formulan preguntas tienen mejor desempeño en comprensión de lectura, que los grupos donde solo pregunta el profesor o donde solo pregunta el estudiante. Sin embargo, Morata Sebastián y Rodríguez Sánchez (1997) ponen de manifiesto las diferencias existentes en el uso de la interrogación didáctica en función de las áreas de conocimiento, -es decir, en las clases de física los estudiantes adoptan un papel más pasivo que los estudiantes de la clase de humanidades- y señalan que la pregunta es utilizada mayoritariamente por los docentes y ampliamente infrautilizada por los alumnos. En contraste Kebede (1999) afirma que no hay relación entre el aprendizaje y la interrogación. Él afirma que las preguntas y las respuestas son útiles en el aprendizaje pero no son suficientes. En el mismo sentido, Fasko y Skidmore (1999) establecieron que la pregunta, independiente de su nivel cognitivo interfiere con el aprendizaje al generar ansiedad en los estudiantes.

Estos mismos autores, Fasko y Skidmore (1999), y Profetto-McGrath et al. (2004) presentan una clasificación de las preguntas según el grado de complejidad: de bajo nivel cognitivo (preguntas de conocimiento o información) y de alto nivel cognitivo (preguntas que requieren procesamiento, análisis, aplicaciones, síntesis, opiniones y sugerencias). Phillips y Duke (2001) y 
Profetto-McGrath et al. (2004) sostienen que la mayoría de las preguntas que formulan los profesores y los estudiantes son de bajo nivel cognitivo, es decir, son preguntas de conocimiento o información. Quizás esta sea una razón para que Kebede (1999) no encuentre alguna relación entre el aprendizaje y la pregunta, porque está visto que el aprendizaje está en función de la complejidad de la pregunta planteada.

Al comprender estos aportes desde el horizonte de la pregunta como una posibilidad para la traducción de saberes a nivel superior, surge entonces la siguiente inquietud: ¿qué tipo de preguntas posibilitaría la traducción? Se sospecha que las preguntas de alto nivel cognitivo son las que posibilitan la traducción, en la medida en que con estas preguntas realmente sucede una comunicación de sentidos de los conocimientos que aprenden los estudiantes, en tanto son preguntas abiertas, porque las preguntas de conocimiento e información (es decir, de bajo nivel cognitivo) no generan muchos espacios para el diálogo. Adicionalmente, si se toma como referente el trabajo de El-Koumy (1997), se abre una posibilidad para pensar en la importancia que tiene el hecho de que el estudiante también genere preguntas en el proceso de aprendizaje, porque en esta medida el conocimiento adquiere un significado para él, además de que su proceso de aprendizaje sería consciente y controlado por él mismo, favoreciéndose de esta manera el autoaprendizaje.

Una segunda perspectiva que se identifica en la investigación en torno a la pregunta, son los trabajos que se ocupan de algunas estrategias didácticas basadas en ella. En estos trabajos se plantea que estrategias didácticas centradas en el estudiante, como la vida en la letras (GONZÁLEZ, 1997), la estética como fuente del conocimiento (GONZÁLEZ, 1999), el proyecto de aula (CARDONA SALAZAR, 2005), el aprendizaje basado en problemas (FASCE et al., 2001 y HERNÁNDEZ TRASOBARES; LACUESTA GILABERTE, 2007), los escenarios (ABRANDT DAHLGREN; ÖBERG, 2001), el seminario (LIN; GORRELL; PORTER, 1999) y la discusión on-line (WHIPP, 2003), sirven como punto de partida para procesos de aprendizaje o como una posibilidad de articular e integrar el mundo académico con el mundo de la vida.

En apariencia, la tendencia es estudiar los efectos de algunas estrategias didácticas en el aprendizaje de los estudiantes, dejando de lado, su articulación con la pregunta. Pero si se analiza detenidamente estas estrategias didácticas, será evidente que la pregunta subyace en cada una de ellas. De modo que al valorar el efecto que tienen las estrategias didácticas sobre el aprendizaje, se está valorando al mismo tiempo, el efecto que tiene la pregunta en dicho proceso. Lo que sería interesante mirar es el tipo de preguntas que surgen 
(tanto en los profesores como en los estudiantes) en el marco de estas estrategias didácticas. De las investigaciones disponibles, Abrandt Dahlgren y Öberg (2001) muestran que con el empleo de los escenarios, entre los estudiantes, se generan preguntas de tipo enciclopédica, de significado, de relación, valorativa y orientada a la solución, siendo las dos primeras las más frecuentes. Por su parte, Whipp (2003) señala que en las discusiones on-line la complejidad de las preguntas de los estudiantes no es muy alta.

Resulta importante resaltar los aportes que hace González en torno a las estrategias didácticas: la vida en la letras (1997) y la estética como fuente de conocimiento (1999), las cuales son productos de procesos investigativos que buscan integrar el mundo de la ciencia y el mundo de la vida y que señala varios tipos de preguntas que posibilitan esta integración. La estrategia la vida en las letras se enmarca dentro del modelo de los procesos conscientes, siendo la pregunta el método de acercamiento entre el docente y el discente, y entre éste y el sistema de signos (conjunto de contenidos objeto de apropiación por parte del estudiante). La estrategia propone tres tipos de preguntas: fácticas, problematológicas y trascendentales. En un sentido complementario, la estrategia la estética como fuente de conocimiento se desarrolla mediante los mismos tres tipos de pregunta, pero mediadas didácticamente por dos construcciones del docente: una metafórica y otra científica.

La pregunta fáctica busca insertar el tema que se va a enseñar con un problema del mundo de la vida. La construcción metafórica busca despertar la mente del estudiante hacia una manera no idéntica, estética, de abordar el conocimiento. La pregunta problematológica pretende relacionar el saber cotidiano y el saber estético con el saber científico. La construcción científica busca enseñar desde la interpretación del docente los conceptos, las leyes y teorías de la ciencia. Y por último la pregunta trascendental o el indicativo de acción busca integrar el mundo de la vida y el mundo de la ciencia, en acciones concretas.

Se presume entonces, que estas estrategias didácticas creadas por González generan en el profesor preguntas de alto nivel cognitivo, lo que conduce a que el estudiante establezca relaciones, analice, compare, interprete y cree cosas desde la comprensión que ha tenido de los contenidos enseñados, derivándose nuevamente relaciones entre la pregunta, el contenido y el método de enseñanza.

Complementariamente, uno de los temas que ocupa la investigación en torno a la pregunta como estrategia didáctica, es la cuestión de la metacognición. Un aspecto que es repetitivo en estas investigaciones es que se resalta la 
consciencia que se tiene de los aprendizajes cuando los estudiantes formulan y encuentran sentido a las preguntas, es decir, cuando las preguntas tienen un horizonte propio. Es así como Lin, Gorrell y Porter (1999) encontraron que a través del seminario y del diario los estudiantes manifiestan cambios conceptuales en torno al tema de estudio, en la medida en que son conscientes de sus preguntas y de las preguntas que se han hecho y que no se han resuelto. Esto lo que pone de manifiesto es que en el proceso de enseñanza el docente debe promover en los estudiantes la elaboración de preguntas y ayudarles en esa construcción, para que de esta manera el estudiante sea más consciente de sus aprendizajes. De lo que se trata entonces, según García Restrepo (2004), es de tener más preguntas que respuestas.

Un asunto que también se deja ver en estas estrategias didácticas es que la pregunta ha sido pensada para alcanzar aprendizajes significativos en los estudiantes, lo cual implica que durante el desarrollo de la estrategia didáctica el estudiante alcance una comprensión de los contenidos por medio de la pregunta, en la medida en que les encuentra sentido y los hace propios. De ahí, que se piense que la estrategia didáctica conduzca al establecimiento de un acuerdo en el estudiante con el fin de posibilitarle la comprensión de contenido, acuerdo que se logra por medio de la pregunta.

Finalmente, en la perspectiva del pensamiento crítico (tercera línea de trabajo), se ubican los trabajos que estudian la interrogación como una estrategia para desarrollar y promover el pensamiento crítico en la educación superior. En este sentido, los estudiantes universitarios deben aprender no solo los conocimientos sino que deben desarrollar habilidades de pensamiento que les permita transferir esos conocimientos al mundo de la vida, siendo la pregunta el medio para desarrollar dichas habilidades. Por ejemplo, Valera Villegasy Madriz (1998) encontraron que los estudiantes al interrogar e interrogarse están conscientes de la existencia de un conocimiento que les toca muy de cerca, de ahí que las preguntas hagan posible la construcción del conocimiento y el desarrollo del pensamiento crítico. Loy, Gelula y Vontver (2004) afirman que los estudiantes que utilizan la pregunta crítica o de alto nivel mejoran sus habilidades de pensamiento crítico. Y Wang (2005) sustenta que la interrogación incrementa las habilidades de pensamiento permitiendo la construcción del conocimiento. No obstante, Arburn y Bethel (1999) y Wang (2001) no encontraron diferencias significativas en cuanto al empleo de la pregunta para desarrollar habilidades de pensamiento crítico. Quizás, lo importante no es la pregunta en sí misma, sino el nivel de complejidad, así como lo indican Loy, Gelula y Vontver (2004). 
Pero ¿qué se entiende por pensamiento crítico? El pensamiento crítico, como lo señalan Hernández y Alvarado (2003) es una operación que no se limita a movilizarse libremente entre las cosas, a detenerse por azar en algunas de ellas o a registrar que las cosas indican algo, sino la operación que establece el nexo entre lo que ellas sugieren y la posibilidad de que esa sugerencia se realice y también que pueda remitir a otra. Es decir, es un proceso que establece relaciones entre los sentidos que portan las cosas y que crea la posibilidad de transmitir esos sentidos a cosas nuevas, como una manifestación de la fusión de horizontes, como una manifestación de la comprensión. Así, el pensamiento crítico es comprensivo y creativo. Surge entonces una inquietud: ¿será que el pensamiento crítico se puede entender como traducción? Es posible. Más aún cuando el concepto crítica se entendió en el renacimiento como un instrumento para encontrar el texto auténtico y original de las traducciones, es decir, un instrumento para encontrar el sentido a los textos. De modo que si el pensamiento crítico se promueve por medio de la pregunta, es probable que la traducción sea posible, igualmente, por medio de la pregunta.

De otro lado, como lo señalan Guzmán Silva y Sánchez Escobado (2006), citando a Hannel y Hannel (1998), el pensamiento crítico requiere de habilidades cognitivas como: a) mirar la información, etiquetarla e identificar hechos; b) comparar, relacionar, hacer analogías; c) clasificar, integrar, encontrar relaciones; d) decodificar, deducir; e) codificar; f) inferir, proyectar, aplicar; g) sintetizar. Se interpreta entonces que estas son las habilidades que se desarrollarían mediante la secuencia de preguntas en el proceso de traducción de los contenidos.

Una categoría muy relacionada con el pensamiento crítico es la comprensión. En el marco de la Línea de Enseñanza para la Comprensión dentro del Proyecto Cero de la Universidad de Harvad, Giordano y Moyano (2003) afirman que es posible enseñar para la comprensión en el nivel superior, es decir, enseñar para pensar y actuar con flexibilidad a partir de lo que se sabe. La parte central del aprendizaje para la comprensión debe ser la realización de actividades de comprensión como: relacionar, justificar, operar, aplicar, comparar, describir, diferenciar, relatar, diagramar, adecuar, analizar, decidir, representar, organizar, secuenciar, conjeturar, reflexionar y diseñar. Es decir, estas actividades son las que debe promover el docente mediante la secuencia de preguntas para posibilitar al estudiante la comprensión de los contenidos.

¿Pero qué guardan en sí este conjunto de habilidades de pensamiento? Al parecer hay una relación con la lógica de construcción de los saberes. ¿Será 
entonces, que la secuencia de preguntas del profesor sigue la lógica del saber que se enseña?

Se observa además, que entre las actividades de comprensión y las habilidades de pensamiento crítico propuestas por Hannel y Hannel existe una asociación, lo que lleva a establecer una relación directa entre la comprensión y el pensamiento crítico. Quizás se trate de una relación dialéctica, donde la comprensión de las cosas determina el pensamiento crítico y a su vez, el pensamiento crítico determina la comprensión de las cosas. Relaciones dialécticas mediadas por la pregunta.

Un sentido que se devela de las investigaciones que se ocupan de la pregunta como una estrategia para desarrollar el pensamiento crítico, es que no se trata solo de la pregunta que formula el profesor a los estudiantes, sino que también se trata de la pregunta que hacen los estudiantes al profesor y que se hacen profesores y estudiantes a sí mismos. Por eso es importante retomar el aporte que hacen El-Koumy (1997) y Valera Villegas y Madriz (1998) donde se reconoce la importancia que tiene el preguntarse a sí mismo en el desarro110 del pensamiento crítico, esto es, "identificar y desafiar suposiciones y explorar e imaginar alternativas" (BROOKFIELD, 1986, citado por VALERA VILLEGAS; MADRIZ, 1998, p. 5). Pensamiento este que implica un pensamiento reflexivo, la necesidad de suspender juicios y el pensar para sí mismo, por sí mismo y con el otro. Dicen Valera Villegas y Madriz que la formación de un pensamiento crítico en el aula implica la oferta de oportunidades para que el estudiante aprenda a indagar y a cuestionar.

Esta oferta de oportunidades para que el estudiante aprenda a preguntar y a preguntarse a sí mismo está determinada por la formación docente. Guzmán Silva y Sánchez Escobado (2006) señalan que la formación del profesor es un aspecto fundamental para facilitar el desarrollo de las habilidades de pensamiento de orden superior. Existe una mejora general en las destrezas de pensamiento crítico en los estudiantes que tomaron cursos con profesores que habían hecho la Especialización en Liderazgo Docente en comparación con los estudiantes de profesores que no habían tomado dicho programa. En este sentido, es importante que el profesor tenga un conocimiento en pedagogía y didáctica para que su práctica sea más consciente e intencional en el desarro1 lo del pensamiento crítico.

Ligado a lo anterior, se percibe recientemente un interés en investigar la doble formación que deben tener los profesores universitarios: de un lado, la formación en un saber específico y de otro lado, la formación pedagógica y didáctica. Estas cuestiones han sido estudiadas por Barajas Perea (2006) y 
Castilla Luna y López de Mesa (2007), quienes plantean como problemática la relación que establece el profesor universitario entre la ciencia que enseña y la manera de enseñarla. La visión que cada profesor tiene sobre los objetivos, procesos, construcción del conocimiento e implicaciones de la ciencia en la sociedad, influye en lo que enseña y cómo lo enseña. Tal vez, esto pueda interpretarse como el dominio que se requiere en la traducción, de la lengua de salida y de llegada.

Esta situación lo que pone de manifiesto es que el uso de la pregunta para desarrollar habilidades de pensamiento no se hace en el vacío, sino que por el contrario se hace en un contexto científico o artístico y además, didáctico, razón por la cual la pregunta está cargada de sentidos propios del saber que se enseña y de la didáctica para posibilitar el entendimiento y comprensión del contenido.

Entonces, pensando nuevamente en la pregunta como una mediación didáctica para la traducción de saberes a nivel superior, los aportes de estas investigaciones son un indicio para sospechar de la pregunta como una posibilidad para la traducción de saberes, en tanto permite comprender -a través de la estrategia didáctica- los sentidos que tienen los saberes y proyectarlos en construcciones nuevas. Como dice García "preguntar es empezar a crear" (GARCÍA RESTREDO, 2004, p. 21).

Tal cual se anunciaba inicialmente, los límites de estas tres perspectivas no son claros, en la medida en que los caminos por los cuales transita una tendencia, se cruzan con los caminos por los cuales transita la otra. Pensar en una relación entre estas categorías, supone considerar la tercera categoría como la de orden mayor, en el sentido que abarca las dos anteriores. Es decir, se reconoce que el desarrollo del pensamiento debe ser la finalidad de la educación superior y una manera para desarrollarlo es la pregunta, en tanto medio, mediación y comunicación. Y a la vez, la pregunta está inmersa y hace parte constitutiva de la estrategia didáctica, entendida ésta como la acción o conjunto de acciones deliberadas que lleva a cabo el profesor en la clase para alcanzar un objetivo de formación. Por eso, se afirma que la pregunta es una estrategia didáctica, porque es una acción deliberada y también es una mediación, porque posibilita alcanzar un objetivo.

Pero además de habitar en la pregunta los sentidos de medio, mediación y comunicación, también habitan los sentidos de contenido y método, porque la pregunta no se da en el vacío, sino que se hace en el contexto de un saber disciplinar y didáctico y en el marco de una estrategia didáctica, de modo que la pregunta se constituye en un camino para alcanzar un objetivo de formación. 
En síntesis, se comprende la pregunta como un medio que porta el sentido de los saberes científicos, artísticos y didácticos. Las preguntas que portan únicamente el sentido de los saberes científicos y artísticos son preguntas propias del preguntar y preguntarse de los investigadores y de los estudiantes frente al saber. Pero las preguntas además, pueden portar el sentido del saber didáctico, siendo éstas las preguntas propias del preguntar y preguntarse del profesor frente al saber que enseña. Dado que la pregunta porta sentidos, la pregunta se considera una estrategia ya que tiene una intención y si estos sentidos son llevados didácticamente a través del método, se considera entonces, una estrategia didáctica.

En segundo lugar, la pregunta es para pensar críticamente, es decir, para develar el sentido de las cosas (el sentido del contenido) y a partir de ello hacer nuestras propias creaciones. Es en este momento donde la pregunta se constituye en una mediación, ya que su actividad o procesos de pensamiento que genera, conduce a nuevas comprensiones. De este modo, son las preguntas de alto nivel cognitivo las que posibilitan hacer esta mediación, en tanto provocan el análisis, la comprensión, la interpretación y la síntesis de las cosas.

$\mathrm{Y}$ en tercer lugar, las estrategias didácticas introducen las preguntas de alto nivel cognitivo para desarrollar las habilidades de pensamiento, pero también estas preguntas son para motivar al estudiante para que se pregunte y recorra el camino hacia el conocimiento, suscitando en el estudiante procesos de aprendizajes conscientes. Así, la estrategia didáctica se desarrolla en el marco de la comunicación, donde profesores y estudiantes comunican los sentidos de las cosas, generando sus propias comprensiones, es decir, sus propios aprendizajes.

Entonces, lo que parece develar este estado en cuestión, es que la pregunta al guardar los sentidos de medio, mediación y comunicación, configura de un cierto modo la relación entre los contenidos que se enseñan y el método empleado para enseñarlos. ¿Será esta configuración la traducción de saberes?

\section{REFERENCIAS}

ABRANDT DAHLGREN, Madeleine; ÖBERG, Gunilla. Questioning to learn and learning to question: Structure and function of problem-based learning scenarios in environmental science education. Higher Education, Amsterdam, v. 41, n. 3, p. 263-282, 2001. 
ARBURN, Theresa M.; BETHEL, Lowell M. Teaching strategies designed to assist community College Science Students' critical thinking. 1999. Disponible en: $<$ http://eric.ed.gov/?q=ED443663\&id=ED443663>. Acceso en: 20 de 2008.

BARAJAS PEREA, Dary Stella. Las relaciones que establece el profesor universitario de biología con la disciplina que enseña. Una explicación epistemológica en dos estudios de caso en la Universidad Tecnológica del Chocó "Diego Luis Córdoba". 2006. 123 f. Tesis (Maestría) - Universidad de Antioquia, Medellín, 2006.

CARDONA SALAZAR, Blanca Lucía. El proyecto de aula como estrategia didáctica para el desarrollo de competencias en la enseñanza de la ingeniería: propuesta y aplicación en la asignatura mecánica de fluidos en la Corporación Universitaria Lasallista. 2005. 79 f. Tesis (Monografía) Universidad de Antioquia, Medellín, 2005.

CASTILLA LUNA, Mónica; LÓPEZ DE MESA, Clara. Los roles del docente en la educación médica. Educación y Educadores, Chía, Colombia, v. 1, n. 10, p. 105-113, 2007.

EL-KOUMY, Abdel Salam A. Effects of three questioning strategies on efl reading comprehension. 1997. Disponible en: $<$ http://eric.ed.gov/?q=ED41 1696\&id=ED411696>. Acceso en: 20 de 2008.

FASCE, Eduardo H.; CALDERÓN, María B.; BRAGA, Luis I. Utilización del aprendizaje basado en problemas en la enseñanza de física en estudiantes de medicina. Comparación con enseñanza tradicional. Revista Médica de Chile, Santiago de Chile, v. 9, n. 129, p. 1031-1037, 2001.

FASKO, Daniel; SKIDMORE JR., Ronald. The effects of questions and anxiety on attention, question confidence, and metacognition. 1999. Disponible en: $<$ http://eric.ed.gov/?q=ED435756\&id=ED435756> $>$. Acceso en: 20 de 2008.

GARCÍA RESTREPO, Claudia Patricia. Más allá de la hermenéutica y la mayéutica: el pensamiento creativo. 2004. 90 f. Tesis (Monografía) Universidad de Antioquia, Medellín, 2004.

GONZÁLEZ, Elvia María. La pedagogía de la imaginación: la vida en las letras. Medellín: Universidad de Antioquia, 1997. 
GONZÁLEZ, Elvia María. El lenguaje estético como estrategia de conocimiento. La Gaceta Didáctica, n. 1, p. 3-7, 1999.

GIORDANO, Maria F.; MOYANO, Marta A. La enseñanza para la comprensión en la formación universitaria. Una experiencia de aula. Alternativas: espacio pedagógico, v. 33, n, 08, p. 61-70, 2003.

GUZMÁN SILVA, Susana; SÁNCHEZ ESCOBADO, Pedro. Efectos de un programa de capacitación de profesores en el desarrollo de habilidades de pensamiento crítico en estudiantes universitarios en el sureste de México. [En línea] Revista Electrónica de Investigación Educativa, v. 2, n. 8, 2006. Disponible en: $<$ http://redie.uabc.mx/vol8no2/contenido-guzman. html>. Acceso en: 28 oct. 2007.

HERNÁNDEZ TRASOBARES, Alejandro; LACUESTA GILABERTE, Raquel. Aplicación del aprendizaje basado en problemas (PBL) bajo un enfoque multidisciplinar: una experiencia práctica. In: CONOCIMIENTO, INNOVACIÓN Y EMPRENDEDORES: CAMINO AL FUTURO.

Universidad de Antioquia, p. 30-43, 2007.

HERNÁNDEZ, Adriana del Rocío; ALVARADO, Gloria. Transformación de las prácticas pedagógicas a través de nuevos dispositivos de comunicación y la escritura de textos argumentativos. [Versión electrónica]. Universidades, v. 25, p. 45-54, 2003. Disponible en: <http://redalyc.uaemex.mx/redalyc/src/ inicio/ArtPdfRed.jsp?iCve=37302505> . Acceso en: 10 oct. 2007.

KEBEDE, S. The relationship between uptake and questioning. System, v. 2, n. 27, p. 261-275, 1999.

LIN, Huey-Ling.; GORRELL, Jeffrey; PORTER, Karen. The road to preservice teachers' conceptual change. 1999. Disponible en: $<$ http://eric.ed.g ov/?q=ED436508\&id=ED436508>. Acceso en: 20 de 2008.

LOY, G. L.; GELULA, M. H.; VONTVER, L. A. Teaching students to question. American Journal of Obstetrics and Gynecology, v. 5, n. 191, p. 1752-1756, 2004. Universidad de Antioquia, 2008.

MORATA SEBASTIÁN, Rosario; RODRÍGUEZ SÁNCHEZ, Manuel. La interrogación como recurso didáctico. Análisis del uso de la pregunta didáctica practicado en dos áreas de conocimiento en el nivel de Formación Profesional. Didáctica, v, 9, p. 153 - 170, 1997. Universidad de Antioquia, 2007. 
PHILLIPS, N.; DUKE, M. The questioning skills of clinical teachers and preceptors: a comparative study. Journal of Advanced Nursing, v. 4, n. 33, p. 523-529, 2001. Universidad de Antioquia, 2008.

PROFETTO-MCGRATH, Joanne; BULMER SMITH, Karen; DAY, Rene A.; YONGE, Olive. The questioning skills of tutors and students in a context based baccalaureate nursing program. Nurse Education Today, v. 5, n. 24, p. 363-372, 2004. Universidad de Antioquia, 2008.

VALERA VILLEGAS, Gregorio; MADRIZ, Gladys. La pregunta en la enseñanza de las ciencias humanas. Un estudio ecológico de aula universitaria. OEI - Revista Iberoamericana de Educación, 1998. Disponible en: <www.rieoei.org/deloslectores/034Madriz.PDF>. Acceso en: 22 mar.2008.

WANG, Ch. Questioning skills facilitate online synchronous discussions. Journal of Computer Assisted Learning, v. 4, n. 21, p. 303-313, 2005. Universidad de Antioquia, 2008.

WANG, Weimin. The relative effectiveness of structured questions and summarizing on near and far transfer tasks. 2001. Disponible en: $<$ http://eric. ed.gov/?q=ED470123\&id=ED470123>. Acceso en: 22 mar.2008.

WHIPP, J. L. Scaffolding critical reflection in online discussions - Helping prospective teachers think deeply about field experiences in urban schools. Journal of Teacher Education, v. 4, n. 54, p. 321-333, 2003. Universidad de Antioquia, 2008. 